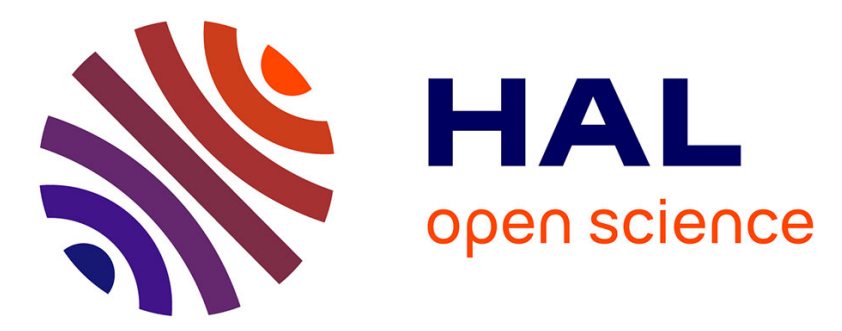

\title{
Data analysis in pulsed-laser, time-resolved photoacoustics: evaluation of errors distribution and confidence intervals
}

\author{
G. Campani, C. Viappiani
}

\section{- To cite this version:}

G. Campani, C. Viappiani. Data analysis in pulsed-laser, time-resolved photoacoustics: evaluation of errors distribution and confidence intervals. Journal de Physique IV Proceedings, 1994, 04 (C7), pp.C7-39-C7-42. 10.1051/jp4:1994710 . jpa-00253151

\section{HAL Id: jpa-00253151 \\ https://hal.science/jpa-00253151}

Submitted on 1 Jan 1994

HAL is a multi-disciplinary open access archive for the deposit and dissemination of scientific research documents, whether they are published or not. The documents may come from teaching and research institutions in France or abroad, or from public or private research centers.
L'archive ouverte pluridisciplinaire HAL, est destinée au dépôt et à la diffusion de documents scientifiques de niveau recherche, publiés ou non, émanant des établissements d'enseignement et de recherche français ou étrangers, des laboratoires publics ou privés. 


\title{
Data analysis in pulsed-laser, time-resolved photoacoustics: evaluation of errors distribution and confidence intervals
}

\author{
G. Campani and C. Viappiani \\ Department of Physics, University of Parma, viale delle Scienze, 43100 Parma, Italy
}

\begin{abstract}
Real time deconvolution of photoacoustic waveforms using nonlinear least squares methods has been successfully used to analyze the temporal profile of the heat released by molecular excited states. In the present work we investigate the statistical distribution of the errors by which the photoacoustic signal is affected. We introduce the $\chi^{2}$ test to judge the goodness of fitting and obtain the confidence intervals of the recovered parameters.
\end{abstract}

\section{INTRODUCTION}

The analysis of the temporal profile of the acoustic waves generated in an absorbing solution by a pulsed laser and detected by an ultrasonic transducer can give information on the kinetics of the nonradiative decays of the excited states and the volume changes induced in the medium. The time dependent heat release has been successfully examined in recent years by means of deconvolution techniques [1]. The waveform of a sample compound is compared to the waveform of a reference compound, i.e. a sample undergoing very fast nonradiative deexcitation with unit efficiency, which reproduces the transfer function of the system. The transfer function is numerically convolved with a heat decay function, usually a sum of exponentials, and compared to the measured sample waveform. The problem of finding the most probable values for the fitting parameters is solved by determining the values corresponding to the minimum in the sum of the squares of the residuals [2], [3]. This methodology requires that some conditions are met [4]: 1) only the dependent variable is affected by errors, 2) the experimental uncertainty on the variable is represented by a gaussian distribution, 3) no systematic errors exist in the data, 4) the functional form of the fitting function is correct and 5) there are enough data points to allow a good sampling. Since the error distribution is not known a priori, non weighted residuals have been used so far in the fitting procedure.

In this work we have determined experimentally the errors distribution of photoacoustic signals and verified that it closely follows a gaussian profile. The knowledge of the errors on the experimental data allows the use of the $\chi^{2}$ test, which gives a statistically meaningful check on the goodness of the fitting. The numerical accuracy of the fitting algorithm has been established by determining the confidence intervals for the fitted parameters and the cross correlation between them [2]. Some comments are done on the presence of systematic errors in the data.

\section{EXPERIMENTAL}

\subsection{Materials}

All chemicals were used as received with no further purification. All measurements were performed at room temperature. Ferrocene was from Sigma, eosin was from Carlo Erba and bromocresol purple was from Eastman Kodak.

\subsection{Photoacoustic measurements}

\subsubsection{Instrumentation}

The photoacoustic instrument has been previously described [5]. Essentially, it consists of an excimer pumped dye laser (Lambda Physik, model EMG50 XeCl excimer laser, FL3100 dye laser, 5ns 
pulse width) whose output is incident on a fused silica absorption cuvette of $1 \mathrm{~cm}$ pathlength. A fraction of the incident light is diverted by a beam splitter to an energy meter (Laser Precision RjP-735 pyroelectric probe, RjP-7620 energy ratiometer) which monitors the energy actually incident on the cuvette. The diameter of the beam inside the cuvette is about $0.5 \mathrm{~mm}$. The linearity of the photoacoustic response with pulse energy was checked and the measurements were carried out in the linear response region. The heat deposited by the photoinitiated events is detected by a Panametrics V103, 1-MHz PZTbased piezoelectric transducer, clamped to the side of the cuvette with a layer of silicone grease interfacing transducer and cuvette. The transducer signal is amplified (Panametrics ultrasonic preamplifier, $60 \mathrm{~dB}$ gain, $0.5-5 \mathrm{MHz}$ bandwidth) and digitized by a digital oscilloscope (LeCroy $9450 \mathrm{~A}$ $400 \mathrm{Ms} / \mathrm{s}$, 8 -bit vertical resolution, $350 \mathrm{MHz}$ bandwidth).

\subsubsection{Data acquisition and preprocessing}

Both the energy meter and the oscilloscope are interfaced by means of a GPIB-IEEE 488 parallel interface to a PS2/50 IBM personal computer that performs both the remote control of the instruments and the data analysis. In order to reduce the noise, data from several laser pulses are averaged by the digital oscilloscope to give one photoacoustic waveform. The energy of each laser shot is transferred to the PC that performs the corresponding average. Usually a 2,000 points waveform is collected and up to 1,000 waveforms can be averaged by the digital oscilloscope. The determination of the standard deviation for each data point is performed on a subset of 100 waveforms. A first preprocessing of the data is required prior to the deconvolution analysis [6]; the baseline is subtracted to the signal, which is then divided by the average laser pulse energy $E$ and by the factor $\left(1-10^{-} \mathrm{A}\right)$, where $A$ is the sample absorbance at the excitation wavelength. Absorbance of each sample was kept close to 0.1 at the excitation wavelength.

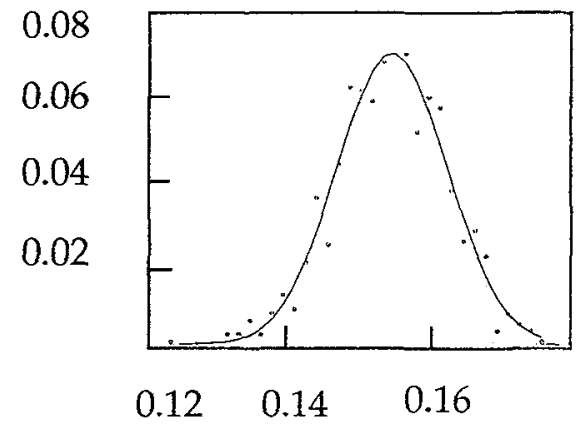

PA signal
Figure 1 Distribution of signal values at the first positive maximum. Solid line is the best fit to the distribution with a gaussian profile.

\subsubsection{Deconvolution algorithm}

The deconvolution algorithm we used for photoacoustic waveforms is based on the parabolic interpolation between three adjoining points [3]. The non linear fitting is performed by means of the Marquardt algorithm [2] and uses the $\chi^{2}$ test. The standard deviation determined experimentally for each channel is used as weighting function in the minimization. The diagonal elements of the error matrix in the proximity of the minimum of the $\chi^{2}$ hypersurface give the confidence intervals of the recovered parameters [2]. The error matrix allows the calculation of the cross correlation coefficients of the recovered parameters as well [4]. The heat decay function we used is a sum of exponentials [3].

\section{RESULTS}

\subsection{Determination of errors distribution}

The errors distribution was determined experimentally by acquiring a set of waveforms and performing the usual statistical calculations on each channel. The acquisition of the statistical set of waveforms was made through a computer program that allowed the acquisition of 1,000 waveforms each composed of 50 data points, along with the corresponding laser pulse energies. The digitized waveforms were written to a $(51 \mathrm{X} 1,000)$ matrix that was successively used for the statistical calculations. The distribution of errors follows a gaussian profile independent of the channel number, although the value of the variance depends on the value of the signal. Figure 1 shows the distribution of the signal values at the first positive maximum, along with the best fit to a gaussian shape. The variance of the dispersion is independent of the number of acquisitions that are averaged. The noise showed a dependence on the absolute value of the signal. For strong signals, as is the case with solutions in ethanol or acetonitrile [7], the noise is mainly determined by the laser instability and the value of the variance for each data is a function of the channel number. In aqueous solutions the noise is essentially independent of the channel number and simply reflects the level of the electrical noise in the apparatus. Figure 2 (bottom) shows the shape of noise for ferrocene in acetonitrile along with the actual photoacoustic waveform. It is evident that the level of electrical noise is about $1 \%$ of the peak value of the signal and for the channels of higher signal amplitude the noise standard deviation is related to the value of the signal. A careful analysis of this shape (not reported) showed that the noise standard deviation can be written as the sum of three terms: 1) a constant term, independent of 
the value of the signal, of the order of magnitude of the electrical noise; 2) a term proportional to the absolute value of the signal and 3) a term proportional to the time derivative of the signal.

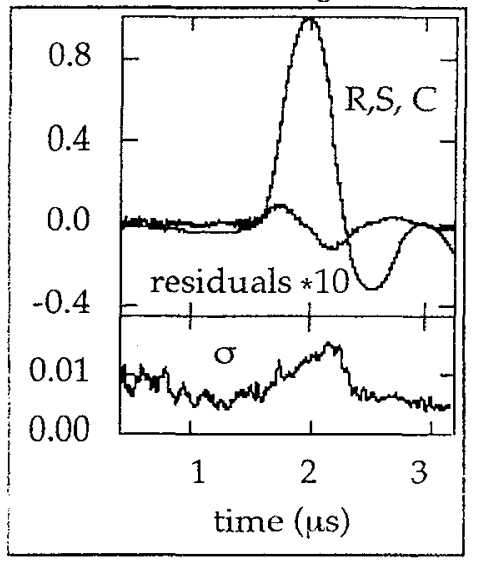

Figure 2 Photoacoustic signal of ferrocene in acetonitrile. Two waveforms were separately acquired and the second one used as a sample waveform. The convoluted waveform was then calculated. Although the signals are perfectly overlapping, the residuals show a systematic deviation, discussed in the text. The lower part of the figure reports the standard deviation of the signal versus channel number determined on a subset of 100 sample waveforms.

\subsection{Numerical simulations}

The parameter retrieval capability of the deconvolution program has been tested with synthetic waveforms obtained from actual waveforms [3]. The synthetic sample waveform was obtained by convolving an exponential heat decay to a reference waveform, and a different reference waveform was then used as response function in order to avoid the same pattern of noise in sample and reference signals. A series of monoexponential decays were used to map the range the deconvolution algorithm has access to. Roughly speaking, lifetimes as short as 50 ns are determined fairly precisely while the lowest $\alpha$ value (0.2) used was retrieved very precisely in all of the situations examined. The longest lifetime accessible was in the range of 5 us.

\subsection{Eosin in aqueous solution}

Measurements were carried out on eosin in air saturated $10 \mathrm{mM}$ phosphate buffer at $\mathrm{pH} 7$ in order to check the statistical test on a well known system. Upon photoexcitation at $480 \mathrm{~nm}$ eosin can either undergo intersystem crossing to a triplet state of energy $190 \mathrm{~kJ} / \mathrm{mol}$ with $\Phi_{I S C}=0.43$ or fluoresce with $\Phi_{\mathrm{f}}=0.2$ [8]. The lifetime of the triplet state of eosin is about $30 \mu$ s at room temperature [8]. When molecular oxygen is present in the solution, the triplet state of eosin produces excited singlet oxygen via energy transfer with unit efficiency [9]. The concentration of molecular oxygen in solution at room temperature is in the range of $0.2 \mathrm{mM}$ and this would result in a lifetime of the

triplet of about $2 \mu$ s. Three heat decays would then be expected in this system, the first being very fast receiving contributions by the internal conversion and the intersystem crossing to the triplet, the second being due to the triplet decay and the energy transfer to molecular oxygen. Finally the third decay is due to the nonradiative relaxation of singlet oxygen whose lifetime is about $3 \mu \mathrm{s}$. The two long lived species have very similar lifetimes and therefore they are expected to be hardly distinguishable The photoacoustic signal of eosin is shown in figure 3 . The left side of the figure reports the results of a single exponential heat decay deconvolution. It is evident that the accordance between sample and reconvoluted waveform is poor, and the $\chi^{2}$ is correspondingly higher than 1 . The residuals show a very strong correlation indicating a systematic deviation between the experimental data and fitted waveform. Fitting with a double exponential strongly improves the visual overlap between the waveforms and results in much less correlated residuals and a $\chi^{2}$ approaching unity. Adding a third exponential decay results in a very slight improvement in $\chi^{2}$ whereas almost no gain is evident from the visual analysis of the residuals (not shown). The statistical weighting of the residuals seems to be correct since the convergence to the minimum is accomplished at the proper value of $\chi^{2}$. Even if the improvement in the fitting with two exponential decays is striking, the remaining correlation of the residuals is not negligible. A first source of non random residuals has been attributed to the numerical approximations involved in the calculation of the convolution. The parabolic approximation in fact fails to reproduce accurately the portions of the waveform that have an inflection point. As it is evident from figure 2 , the highest residuals for the reconvolution of a reference waveform are found for the channels corresponding to these points. A second source of non random errors is possibly due to the presence of acoustical reflections inside the cuvette, whose amplitude is different for the sample and reference waveform. The long time tail of the signal shows in fact a marked deviation from the convoluted waveform, possibly reflecting the arrival of the acoustical reflections. The autocorrelation function (top of figure 3 ) reflects these systematic errors and doesn't drop to zero after the first few channels. One of the problems encountered 
with these measurements is the strong correlation between the amplitude and the lifetime of the long lifetime decays that lead to multiple minima on the $\chi^{2}$ hypersurface.

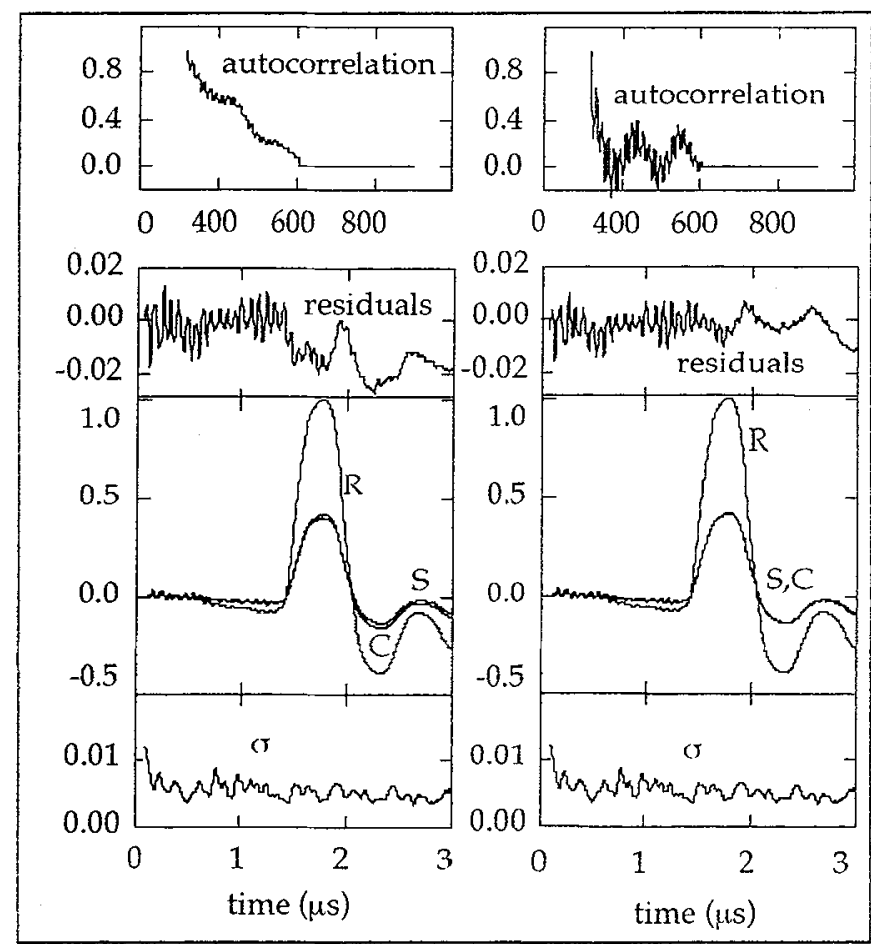

Figure 3 Photoacoustic signal of eosin in $10 \mathrm{mM}$ phosphate buffer at $\mathrm{pH} 7$. The reference waveform was obtained with bromocresol purple. Left. Results of one exponential decay analysis, $\alpha=0.402 \pm 0.0006, \tau=10 \pm 0.3 \mathrm{~ns}, \chi^{2}=7.31$. Right. Results of two exponential decays analysis, $\alpha_{1}=0.399 \pm 210^{-6}$, $\tau_{1}=2 \pm 0.02 \mathrm{~ns}, \alpha_{2}=0.464 \pm 210^{-5}, \tau_{2}=4.96 \pm 410^{-5} \mathrm{~s}, \chi^{2}=1.001$.
In table 1 we report the crosscorrelation matrix for the data shown in figure 3 , that evidences the strong coupling between $\alpha_{2}$ and $\tau_{2}$. Finally we wish to comment on the confidence intervals of the recovered parameters. The value of the errors calculated by means of the diagonal elements are well below the actual experimental accuracy. In fact the usual reproducibility of a measurement in photoacoustics is of the order of $5-10 \%$, while the confidence intervais indicate errors a couple of orders of magnitude smaller. The errors determined by the numerical analysis only refiect the numerical accuracy of the fitting procedure, that is the actual width of the minimum in the $\chi^{2}$ hypersurface and therefore should not be used as an estimate of experimental accuracy which should be determined by performing separate measurements and calculating the standard deviations from these independent acquisitions.

Table 1 Cross correlation coefficients of the recovered parameters

$\left(\begin{array}{ccccc} & \alpha_{1} & \tau_{1} & \alpha_{2} & \tau_{2} \\ \alpha_{1} & 1 & 0.057 & 0.272 & 0.29 \\ \tau_{1} & 0.057 & 1 & -0.055 & 0.045 \\ \alpha_{2} & 0.272 & -0.055 & 1 & 0.97 \\ \tau_{2} & 0.29 & 0.045 & 0.97 & 1\end{array}\right)$

\section{REFERENCES}

1. Rudzki, J. E., J. L. Goodman, and K. S. Peters. 1985. J. Am. Chem. Soc. 107:7849-7854.

2. Bevington, P. R. 1969. Data reduction and error analysis for the physical sciences. McGraw-Hill. New York.

3. Small, J. R. 1992. Deconvolution analysis for pulsed-laser photoacoustics. In Numerical computer methods. L. Brand, and M. L. Johnson, editor. Academic Press, Inc., San Diego. 505-521.

4. Johnson, M. L., and L. M. Faunt. 1992. Parameter estimation by least-squares methods. In Numerical computer methods. L. Brand, and M. L. Johnson, editor. Academic Press, Inc., San Diego. 1-37.

5. Losi, A., R. Bedotti, L. Brancaleon, and C. Viappiani. 1993. J. Photochem. Photobiol. B: Biology. 21:69-76.

6. Small, J. R., L. J. Libertini, and E. W. Small. 1992. Biophys. Chem. 42:24-48.

7. Braslavsky, S. E., and G. E. Heibel. 1992. Chemical Reviews. 92:1381-1410.

8. Carmichael, I., and G. L. Hug. 1989. Spectroscopy and intramolecular photophysics of triplet states. In CRC Handbook of Organic Photochemistry. J. C. Scaiano, editor. CRC Press, Boca Raton, FL. 369403.

9. Chartier, A., J. Georges, and J. M. Mermet. 1990. Chem. Phys. Lett . 171:347-352. 